\title{
Influence of joint rigidity on the elastic buckling load on sway and non-sway steel frames
}

\author{
A. N.T. Ihaddoudène ${ }^{a *}$, M. Saidani ${ }^{b}$, and J. P. Jaspart ${ }^{\mathrm{c}}$ \\ ${ }^{a}$ Faculty of Civil Engineering, University of Science and Technology HouariBoumediene., Algiers, Algeria. \\ bepartment of Civil Engineering, Architecture and Building, Coventry University,England,UK. \\ ${ }^{\mathrm{c}}$ DepartmentArGEnCo, Liège University, 4000 Liège 1, Belgium. \\ *corresponding author, e-mail address: nacer_i@yahoo.fr
}

\begin{abstract}
Much work has been conducted in the past on the influence of the rigidity of structural joints on the behavior of steel frames. Buckling of a column is fundamental to the design of load bearing structures mainly when the analysis of such frames takes into account the effect of the connection flexibility.

The present work deals with such an effect on the elastic buckling load of plane steelframes. The proposed model consists in the development of comprehensive approach taking into account, the effects of the joint rigidity, the elastic buckling load for both sway and nonsway frames. Only one element is required over the length of the element to model stability, which let to solve practical problems with little computational effort. Some practical formulas for determining critical load for plane steel frames are then presented. The elastic buckling load is found to be strongly affected by semi-rigid joints and reveals that the proposed model is computationally very efficient with the expressions presented being general.
\end{abstract}

Keywords:Steel structures; connection flexibility; plane frames; elastic buckling load.

\section{Introduction}

Conventional analysis and the design of steel frames assume either perfectly rigid or pinned joints. However, the real behaviour of the joints is between these two extreme cases; in this intermediate case of semi rigid joints, some rotation with corresponding bending moments will develop between the beam and column elements. The concept of semi rigid joints in steel structures is well accepted as structural benefits of using semi-rigid joints are widely recognized and there is a general agreement to include the beam-column joint deformations in structural analysis.

Significant research has been carried out using mechanical models to study the joint's behaviour and to introduce their effect in the analysis of structures.Simões da Silva [1] proposed a generic model for steel joints under generalized loading. Ihaddoudène [2] presented a mechanical model of the connections, where the rigidity of the joint is represented by means of rotational and translational springs introducing the concept of non deformable element of nodes, thus describing relative displacements and rotations between the nodes and the elements of the structure. Eurocode 3 Part 1-8 refers [3], for the characterization of the joint mechanical response to the component method based on some different researches and amongst them Jaspart [4]. Several authors [5-9] have presented models for determining the effective length factor of a beam-column with end restraints. Ermopoulos [5] presented a model for determining an equivalent buckling length of compression columns with semi rigid joints. Essa [6] proposed a design method for the evaluation of the effective length for columns in unbraced multistory frames. Raftoyiannis [7] presented the effects of the joint flexibility and elastic bracing system on the buckling load. Mageirou and Gantes [8], Gantes and Mageirou [9] proposed a model of an individual column representing a multistory frame where the member contributions converging at the bottom and top ends of the column are represented by equivalent springs. 


\section{Mechanical model}

The mechanical model adopted (Ihaddoudène [2]) is based on the analogy of three springs. A bar element subjected to both a compression axial force $N$ and bending moments $M_{i}$ and $M_{j}$ with semi-rigid joints (Fig. 1 and Fig. 2) at each end, is considered. The modified stiffness matrix including both the effects of axial force and connection flexibility, one needs to consider different situations [10].

In the local reference system, the stiffness matrix which is represented by the nodal degrees of freedom $\left(\bar{V}_{1}, \bar{\theta}_{1}, \bar{V}_{2}, \bar{\theta}_{2}\right)$ of an element is given by: $\quad \overline{K_{e}}=\left[\begin{array}{llll}k_{11} & k_{12} & k_{13} & k_{14} \\ k_{21} & k_{22} & k_{23} & k_{24} \\ k_{31} & k_{32} & k_{33} & k_{34} \\ k_{41} & k_{42} & k_{43} & k_{44}\end{array}\right]$

\subsubsection{Bar element under unit rotation $\theta_{i}=1$}

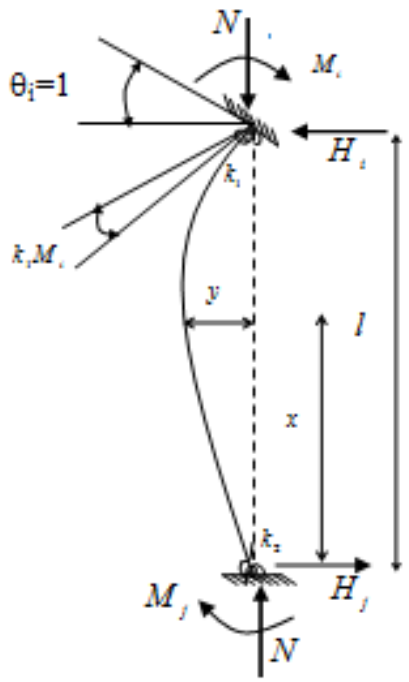

Fig. 1. Bar element subjected to a unit rotation.

Horizontal force equilibrium

$H_{i}=H_{j}=H$

Moment equilibrium at the distance $x$

$$
M(x)=N y+H x-M_{j}
$$

Moment equilibrium at end " $i$ "

$$
M_{i}=H l-M
$$

The equilibrium of this column in its buckled condition is described

$$
\begin{aligned}
& E I y^{\prime \prime}=-N y-H x+M_{j} \\
& y^{\prime \prime}+\alpha^{2} y=-\frac{H x}{E I}+\frac{M_{j}}{E I}
\end{aligned}
$$

Where

$$
\alpha^{2}=\frac{N}{E I}
$$

The general solution of equation (6) is

$$
y(x)=A \sin \alpha x+B \cos \alpha x-\frac{H x}{\alpha^{2} E I}+\frac{M_{j}}{\alpha^{2} E I}
$$

where $A$ and $B$ are the constants of integration to be determined from the boundary conditions for $y(0)=0$ and $y(l)=0$

The solution of the system of equations obtained is given (27) by:

$$
\begin{aligned}
& H=\frac{w}{l} \zeta_{l}(v) \\
& M_{j}=w \phi_{1}(v) \\
& M_{i}=w \phi_{2}(v)
\end{aligned}
$$

In which:

$$
\begin{aligned}
& \zeta_{1}(v)=\frac{v^{2}\left(1-\cos v+k_{2} v w \sin v\right)}{D} \\
& \phi_{1}(v)=\frac{v(v-\sin v)}{D} \\
& \phi_{2}(v)=\zeta_{1}(v)-\phi_{1}(v)
\end{aligned}
$$

Where

$$
\begin{aligned}
& D=(2-2 \cos v-v \sin v)+\xi\left(v, k_{1}, k_{2}\right) \\
& v=\alpha l=l \sqrt{\frac{N}{E I}}
\end{aligned}
$$

And

$$
w=\frac{E I}{l}
$$

A similar procedure is conducted for the bar element of the Fig. 2, the reaction $H$ and the moment equilibrium at the distance $x$ has the same expressions as given respectively by the Eq.(1) and Eq.(2); the expression of the moment $M_{i}$ is given as (Ihaddoudène and Jaspart [11]): 


$$
M_{i}=N+H l-M_{j}
$$

$$
\left[\overline{K_{e}}\right]=E I\left[\begin{array}{c}
\frac{v^{3}(s+\Omega)}{D l^{3}} \\
S Y M
\end{array}\right.
$$

With:

$\Omega=\left(k_{1}+k_{2}\right) v w c-k_{1} k_{2}(v w)^{2} s$

$\eta_{1}=k_{1} v w s$

$\eta_{2}=k_{2} v w s$

"s" and "c" are sin and cos of an angle.

The proposed model which is based on functions accounting for semi-rigid connections and predominant axial load has the advantage of being explicit and simple to solve practical problem with little computational effort. Some practical formulas can be derived from the proposed formulation as reported in the section below.

\section{Practical formulas}

\section{1. "Semi-rigid/pinned" element}

Let us consider the element of Fig. 2 below with semi-rigid at end " $\mathrm{i}$ " and pinned at end " $\mathrm{j}$ ", one can derived the expressions of the functions respectively for unit rotation and unit displacement as:

\subsubsection{Beam element for unit rotation $\theta_{i}=1$}

Parameters in Fig. 2a:

$$
\begin{gathered}
H=\frac{\omega}{l} \zeta_{1}(v) \\
M_{i}=H l=\omega \zeta_{1}(v) \\
\zeta_{l}(v)=\frac{v^{2} \tan v}{\tan v-v+k_{1} v^{2} \omega \tan v}
\end{gathered}
$$

For the particular case of $k_{1}=0$ the function
The entire modified stiffness matrix derived [10] is then as follows:

$$
\left.\begin{array}{cc}
-\frac{v^{3}(s+\Omega)}{D l^{3}} & \frac{v^{2}\left(1-c+\eta_{1}\right)}{D l^{2}} \\
\frac{v^{2}\left(1-c+\eta_{2}\right)}{D l^{2}} & \frac{v(v-s)}{D l} \\
\frac{v^{3}(s+\Omega)}{D l^{3}} & \frac{v^{2}\left(1-c+\eta_{1}\right)}{D l^{2}} \\
& \frac{v\left(s-v c+v \eta_{1}\right)}{D l}
\end{array}\right]
$$

$$
\zeta_{1}(v)=\frac{v^{2} s}{s-v c}=\frac{v^{2} \tan v}{\tan v-v}
$$

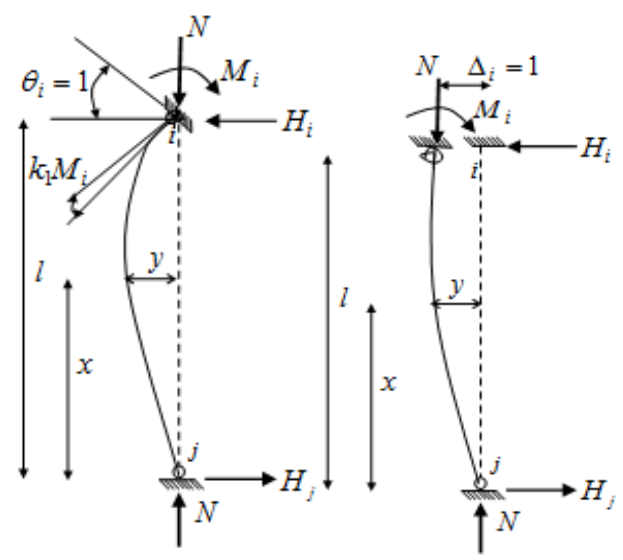

a-Unit rotation $\theta_{i}=1 \mathrm{~b}$ - Unit translation $\Delta_{i}=1$

Fig. 2. "Semi-rigid/pinned" element

\subsubsection{Beam element for unit displacement $\Delta_{i}=1$}

Parameters in Fig. 2b:

$$
\begin{gathered}
H=\frac{\omega}{l^{2}} \zeta_{2}(v) \\
M_{i}=H l+N=\frac{\omega}{l} \phi_{4}(v) \\
\zeta_{2}(v)=\frac{v^{3}\left(1-k_{1} v \omega \tan v\right)}{\left(\tan v-v+k_{1} v^{2} \omega \tan v\right)} \\
\phi_{4}(v)=\frac{v^{2} \tan v}{\tan v-v-k_{1} v^{2} \omega \tan v}
\end{gathered}
$$

Some particular cases can be considered:

$k_{1}=0$ 
$\zeta_{2}(v)=\frac{v^{3} c}{s-v c}=\frac{v^{2}}{\tan v-v}$

$\phi_{4}(v)=\frac{v^{2} s}{s-v c}=\frac{v^{2} \tan v}{\tan v-v}$

$k_{1}=\infty$

$\zeta_{2}(v)=-v^{2}$ and $\phi_{4}(v)=0$

\section{2. "Semi rigid at $i$, fully rigid at $j$ "}

The beam element of Fig. 3 below is considered for unit rotation and unit displacement:

\subsubsection{Beam element for unit rotation $\theta_{i}=1$}

Parameters in Fig. 3a:

$$
\begin{gathered}
H=\frac{\omega}{l} \zeta_{1}(v) \\
M_{i}=\omega \phi_{2}(v) \\
M_{j}=\omega \phi_{1}(v) \\
\zeta_{1}(v)=\frac{v^{2} s(1-c)}{(v-s) \cdot A_{1}+(1-c) \cdot A_{2}} \\
\phi_{1}(v)=\frac{v s(v-s)}{(s-v) \cdot A_{1}+(1-c) \cdot A_{2}} \\
\phi_{2}(v)=\frac{v s(s-v c)}{(s-v) \cdot A_{1}+(1-c) \cdot A_{2}} \\
\hline \text { Where }: A_{1}=1-c+k_{1} v w s \\
A_{2}=s-v c+k_{1} v^{2} w s
\end{gathered}
$$

Particular cases:

For $k_{1}=0$

$\zeta_{1}(v)=\frac{v^{2} s}{2 v-v c-v}$

$\phi_{1}(v)=\frac{v s(v-s)}{(1-c)(2 s-v c-v)}$

$\phi_{2}(v)=\frac{v s(s-v c)}{(1-c)(2 s-v c-v)}$

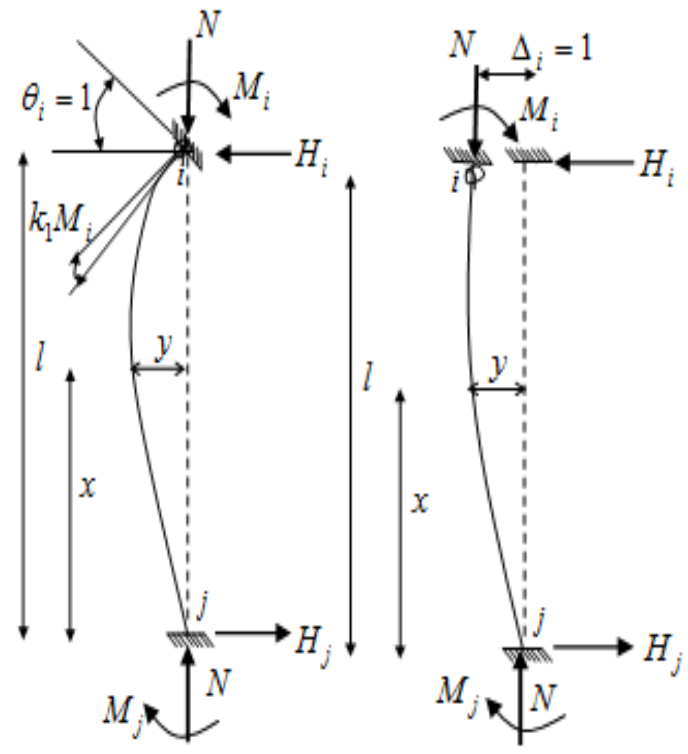

$\mathrm{a}-$ Unit rotation $\theta_{i}=1 \mathrm{~b}-$ Unit translation $\Delta_{i}=1$

Fig. 3. "Semi-rigid/rigid"element.

3.2.2Beam element for unit
displacement $\Delta_{i}=1$

Parameters in Fig. 3b:

$H=\frac{\omega}{l^{2}} \zeta_{2}(v)$
$M_{j}=\frac{\omega}{l} \phi_{3}(v)$
$M_{i}=\frac{\omega}{l} \phi_{4}(v)$
$\zeta_{2}(v)=\frac{v^{3} s\left(s+k_{1} v \omega c\right)}{(s-v)(1-c)+A_{1} \cdot(s-v c)}$
$\phi_{3}(v)=\frac{v^{2} s\left(1-c+k_{1} v \omega c\right)}{(s-v)(1-c)+A_{1} \cdot(s-v c)}$
$\phi_{4}(v)=\frac{v^{2} s(1-c)}{(s-v)(1-c)+A_{1} \cdot(s-v c)}$

\section{3. "Pinned-fully rigid" ends.}

\subsubsection{Beam element for unit rotation $\theta_{i}=1$}

For the element with the pinned-fully rigid ends of the Fig. 4a, the solution of the differential equation gives the expression of the deflection such that:

$y(x)=A \sin \alpha x+B \cos \alpha x-\frac{H x}{\alpha^{2} E I}$ 
By determining the integrating constants $\mathrm{A}$ and $\mathrm{B}$ corresponding to the cases under consideration, we obtain the relation:

$$
H=\frac{3 \omega}{l} \phi_{1}(v)
$$

Where:

$\phi_{1}(v)=\frac{v^{2} s}{3(s-v c)}=\frac{v^{2} \tan v}{3(\tan v-v)}$

$v=\alpha l=l \sqrt{\frac{N}{E I}}$

Hence the bending moment:

$$
M_{i}=H l=3 \omega \phi_{1}(v)
$$

For $v=0($ then for $N=0), \phi_{1}(v)=1$

$$
M_{i}=3 \omega \text { and } H=\frac{3 \omega}{l}
$$

\subsubsection{Beam element for unit displacement $\Delta_{i}=1$}

For the element of the Fig. $4 \mathrm{~b}$ the deflection which has the expression as :

$$
H=\frac{w}{l^{2}} \cdot \frac{v^{3} c}{s-v c}=\frac{3 w}{l^{2}} \eta_{l}(v)
$$

Where

$\eta_{1}(v)=\frac{v^{3} c}{s-v c}=\frac{v^{3}}{3(\tan v-v)}$

The bending moment at node " $i$ "

$$
\begin{aligned}
& M_{i}=\frac{3 w}{l} \eta_{1}(v)+\frac{v^{2} w}{l}=\frac{3 w}{l}\left(\eta_{1}(v)+\frac{v^{2}}{3}\right) \\
& M_{i}=\frac{3 w}{l} \phi_{l}(v)
\end{aligned}
$$

Noting that:

$$
\eta_{1}(v)+\left(\frac{v^{2}}{3}\right)=\frac{v^{2} \operatorname{tg} v}{3(\operatorname{tg} v-v)}=\phi_{1}(v)
$$

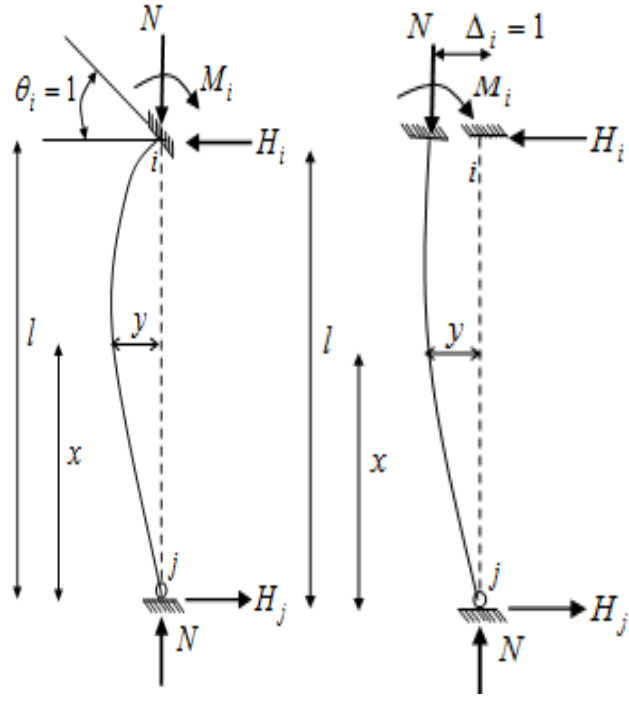

a-Unit rotation $\theta_{i}=1 \quad \mathrm{~b}-\mathrm{Unit}$ translation $\Delta_{i}=1$

Fig. 4. "Rigid- pinned" element.

For $v=0$ which corresponds to $\phi_{1}(0)=1$

$$
M_{i}=\frac{3 w}{l} \text { and } H=\frac{3 w}{l^{2}}
$$

\section{Sway and non-sway frame}

Some examples previously published $[8,9]$ are given, for which the proposed approach is demonstrated and the results are compared and validated. The two situations of sway and non sway frames shown in Fig. 5a and Fig. 5b are considered, respectively. Table 1 and Table 2 give the value of the critical load obtained for these two cases using the different considered methods.

The characteristics of the structural elements are given below:

For the beam $\left\{\begin{array}{l}E I=48573 k N . m^{2} \\ E A=896490 k N\end{array}\right\}$ and for the column $\left\{\begin{array}{l}E I=90699 k N . m^{2} \\ E A=1272600 k N\end{array}\right\}$ and the flexibility $k_{1}=1 / 150 \mathrm{rad} / \mathrm{kN} . \mathrm{m}$ 


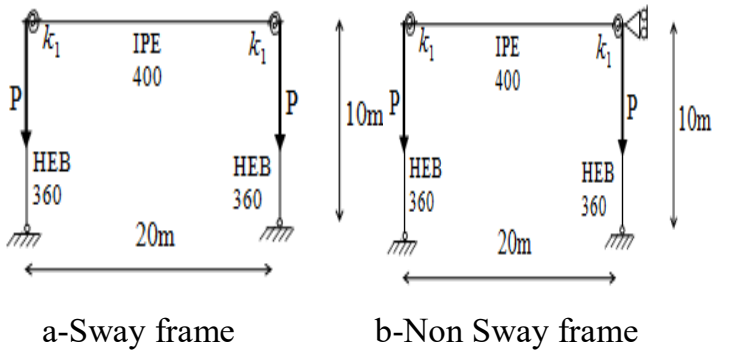

Fig. 5. One story frame [8]

Table 1. Comparison of the critical load values for sway frame.

\begin{tabular}{ccc}
\hline $\begin{array}{c}\text { Methodsuse } \\
\mathbf{d}\end{array}$ & $P_{c r}(k N)$ & $\frac{P_{c r}-P_{c r}, M E F}{P_{c r}, M E F}(\%)$ \\
\hline OSSA2D & 14.76 & 0 \\
{$[12]$} & & 5983.56 \\
EC3 [8] & 898.78 & 0 \\
EC3 clause & 14.76 & 0 \\
5.2.1 (4)B [3] & & \\
Reference [8] & 14.77 & -0.406 \\
Current study & 14.7 & \\
\hline
\end{tabular}

For the sway frame, the critical load obtained by the proposed method is very close to that given by Mageirou et al. [8] and is respectively equal to $P_{c r}=14.7 \mathrm{kN}$ and $P_{c r}=14.77 \mathrm{kN}$ and is in a good agreement with that obtained with the method clause 5.2.1(4)B of EC3 [3].

Table 2. Comparison of the critical load values for non sway frame.

\begin{tabular}{ccc}
\hline $\begin{array}{c}\text { Methods } \\
\text { used }\end{array}$ & $P_{c r}(k N)$ & $\frac{P_{c r}-P_{c r}, M E F}{P_{c r}, M E F}(\%)$ \\
\hline F.E.M- & \\
MSC- & 8980.67 & 0 \\
NASTRA & \\
N [8] & \\
F.E.M - & & \\
OSSA2D & 8739.5 & -2.7 \\
[12] & & \\
\hline
\end{tabular}

\begin{tabular}{ccc}
\hline EC3 $[8]$ & 9980.74 & 11.14 \\
EC3 & & \\
Clause & 8987.5 & 0 \\
$5.2 .1(4) \mathrm{B}$ & & \\
{$[3]$} & & \\
Reference & 8980.67 & 0 \\
{$[8]$} & & \\
Current & 8980.6 & -0.0008 \\
study & & \\
\hline
\end{tabular}

The results as reported in the reference (Gantes and Mageirou [11]) ( See Tables 1 and 2 ) calculated with Eurocode 3 [8] for sway and nonsway frames are very different from those obtained by the authors with EC3 clause 5.2.1(4)B [3].

The results obtained using this analytical formulation are clearly consistent with those obtained by the above references, the finite element method and the application of EC3 clause 5.2.1(4)B for both sway and non sway frames. The formulation provides a simple solution for each of the design situations that refer to the concept of elastic critical resistance.

\section{Conclusions}

A mechanical model for determining the elastic buckling load for both sway and nonsway plane of steel frames with semi-rigid connections was presented and a corresponding practical formula are derived. Only one element is sufficient over the length of the element to model stability. The proposed model which is based on functions accounting for semi-rigid connections and predominant axial load has the advantage of being explicit and simple to solve practical problem with little computational effort. The determination of the critical load is very sensitive to the rigidity of the joint, even when the structure is almost a mechanism; the results obtained from the formulation presented were not affected.The results obtained using the proposed analytical formulation are clearly consistent with those obtained from the literature, for both sway and non-sway frames. 


\section{References}

[1] Simoes da Silva, L. Towards a consistent design approach for steel joints under generalized loading. Journal of Constructional Steel Research, 2008; 68: 1059-1075.

[2] Ihaddoudène ANT. Analyse de la stabilité des structures à assemblages semi-rigides, Ph.D Thesis, Faculty of Civil Engineering, University of Sciences and Technology, U.S.T.H.B, Algeria, 2008.

[3] Eurocode 3: Design of Steel Structures - Part 18: Design of joints, Brussels, CEN, 2005.

[4] Jaspart JP. Design of structural joints in building frames. Progress in Structural Engineering and Materials, 2002; 4 (1):18-34. DOI: 10.1002/pse. 105 .

[5] Ermopoulos J. Buckling length of framed compression members with semi-rigid connections .Journal of Constructional Steel Research 1991;18(2):139-54.

[6] Essa HS: Stability of columns in unbraced frames. Journal of Structural Engineering, Volume 123, 1997; No.7: 952-957.

[7] Raftoyiannis IG. The effect of semi-rigid joints and an elastic bracing system on the buckling load of simple rectangular steel frames. Journal of Constructional Steel Research, 2005; 61: 1205- 1225. DOI: 10.1016/j.jcsr.2005.01.005.

[8] Mageirou GE, Gantes CJ. Buckling strength of multi-storysway, non-sway and partially-sway frames with semi-rigid connections. Journal of Constructional Steel Research, 2006; 62: 893905. DOI: 10.1016/j.jcsr.2005.11.019.
[9] Gantes CJ, Mageirou GE. Improved stiffness distribution factors for evaluation of effective buckling lengths in multi-story sway frames. Engineering Structures, 2005; 27:1113-1124.

[10] Ihaddoudène ANT, Saidani M and Jaspart JP. Mechanical model for determining the critical load of plane frames with semi-rigid joints subjected to static loads. Engineering Structures, 2017; 145:109-117.

[11] Ihaddoudène ANT, Jaspart JP. Stability of non sway steel frames with semi rigid connections,Proceedings of the 14th International Conference on Civil, Structural and Environmental Engineering \& Computing, September 3-6, 2013. Cagliari, Italy.

[12] WinOssa2D, ver.3.31: Elasticdesign of plane frames. University of Liège, Belgium. 